\title{
Wavelength and Phase Detection Based SMS Fiber Sensors Optimized With Etching and Nanodeposition
}

\author{
Yamile Cardona-Maya, Ignacio Del Villar, Abian B. Socorro, Jesus M. Corres, Member, IEEE, \\ Ignacio R. Matias, Senior Member, IEEE, and Juan F. Botero-Cadavid
}

\begin{abstract}
The development of an optical fiber refractometer by hydrogen fluoride etching and sputtering deposition of a thin-film of indium tin oxide on a single-mode-multimode-single-mode fiber structure has been analyzed with the aim of improving the sensitivity to the changes of the refractive index (RI) of the external medium. The device is sensitive to the RI changes of the surrounding medium, which can be monitored by tracking the spectral changes of an attenuation band or with a fast Fourier transform (FFT) analysis. By using an optical spectrum analyzer combined with a simple FFT measurement technique, the simultaneous realtime monitoring is achieved. The results show that the sensitivity depends on the thin-film thickness. A maximum of $1442 \mathrm{~nm} / \mathrm{RIU}$ (refractive index unit) in the 1.32-1.35 RIU range has been attained. In addition, a theoretical analysis has been performed, where simulations matched with the experimental results. As a practical application of the developed optical fiber structure, $\mathrm{a}^{\circ} \mathrm{Brix}\left({ }^{\circ} \mathrm{Bx}\right)$ sensor has been implemented with a sensitivity of $2.13 \mathrm{~nm} /{ }^{\circ} \mathrm{Bx}$ and 0.25 $\mathrm{rad} /{ }^{\circ} \mathrm{Bx}$ respectively for wavelength and phase shift detection.
\end{abstract}

Index Terms-Etching, optical fiber sensor, refractive index, single-mode-multimode-single-mode (SMS), thin-films.

\section{$\mathbf{O}$}

\section{INTRODUCTION} due their multiple advantages such as compact size and high resolution. They can be used in harsh environments and allow for minimally invasive procedures to be performed [1].

Up until now, several technologies have been used to develop optical fiber refractometers. Some of these technologies include: fiber gratings [2], [3], long period fiber gratings [4], resonances [5], [6], evanescent field [7] and interferometers [8].

Manuscript received April 21, 2017; revised June 5, 2017; accepted June 16, 2017. This work was supported in part by the Agencia Estatal de Investigación, in part by Fondo Europeo de Desarrollo Regional (TEC2016-78047-R), in part by the Government of Navarre through its projects with references: 2016/PI008, 2016/PC025, and 2016/PC026, and in part by the Colombian Administrative Department of Science, Technology and Innovation-Colciencias, through the Program for national doctorates, calling 617 of 2013. (Corresponding author: Yamile Cardona-Maya.)

Y. Cardona-Maya and J. F. Botero-Cadavid are with the School of Physics, Universidad Nacional de Colombia, Medellin 050034, Colombia (e-mail: ycardon@unal.edu.co; jfbotero@unal.edu.co).

I. Del Villar, A. B. Socorro, J. M. Corres, and I. R. Matias are with the Department of Electrical and Electronic Engineering, Institute of Smart Cities, Public University of Navarre, Pamplona 31006, Spain (e-mail: ignacio.delvillar@unavarra.es; ab.socorro@unavarra.es; jmcorres@unavarra. es; natxo@unavarra.es).

Color versions of one or more of the figures in this paper are available online at http://ieeexplore.ieee.org.

Digital Object Identifier 10.1109/JLT.2017.2719923
Within the last group, a single-mode-multimode-single-mode SMS fiber refractometer has been studied and a sensitivity of $1199.8 \mathrm{~nm} /$ RIU for the range of refractive indices from 1.321 to 1.382 has been reported [9]. In this work, the experimental and theoretical demonstration of a novel and high-sensitivity refractometric sensor is reported. The developed sensor relies on multimodal interference in etched SMS fiber structures coated with a thin-film that improves the previously sensitivity reported with this structure [9]. Moreover, by application of fast Fourier transform analysis (FFT), it is possible to use both wavelength and phase monitoring of the parameter to detect.

The proposed SMS configuration of this work consists of input and output single-mode fibers (SMFs) that are spliced to a section of a multimode coreless fiber (MMF) of a certain length. The operation mechanism of this refractometer is based on the multimode interference (MMI). When the light propagating along the input SMF enters the MMF section, several eigenmodes of the MMF are excited and interference among different modes occurs during the propagation along the MMF section. Finally, the light is coupled into the output SMF at the end of the MMF section [10]. Through the reduction of the diameter in the MMF section, the evanescent field penetrates further into the surrounding medium, thus increasing the sensitivity [11], [12]. Furthermore, a deposited high refractive index thin-film enhances the interaction with the environment surrounding the fiber and also permits to increase the sensitivity.

To sum up, the enhancement of the sensitivity in the SMS configuration in this work is achieved by gathering two different phenomena, diameter reduction and thin-film deposition.

\section{Methods AND MAterials}

Coreless MMF segments from POFC Inc. (Taiwan) and standard SMF pigtails from Telnet Redes Inteligentes Inc. (Zaragoza, Spain) were used in this study. The SMS structure consists on a 14-mm segment of coreless MMF spliced on each end to standard SMF pigtails, as shown in Fig. 1(a). This structure was etched using hydrofluoric acid $40 \%$ (HF) until the diameter of the fiber was reduced to approximately $25 \mu \mathrm{m}$ [see Fig. 1(b)]. This process took 70 minutes. Finally, a thin-film of Indium Tin Oxide (ITO) was deposited by sputtering on the etched region [see Fig. 1(c)]. The effect of the etching and the deposition was studied theoretically and experimentally.

The theoretical analysis was performed with FIMMWAVE. The propagation was obtained with FIMMPROP, a module in- 


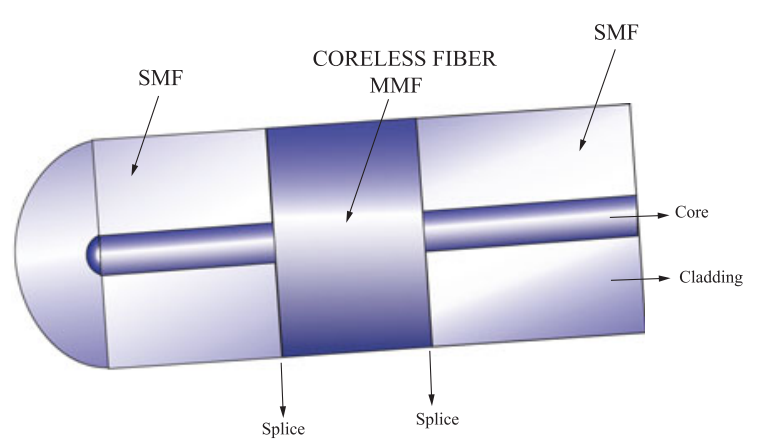

(a)

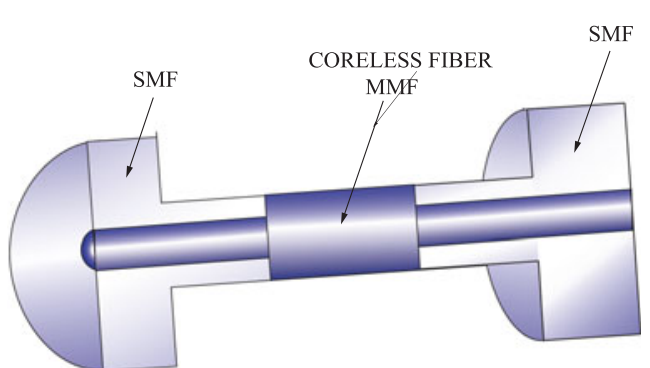

(b)

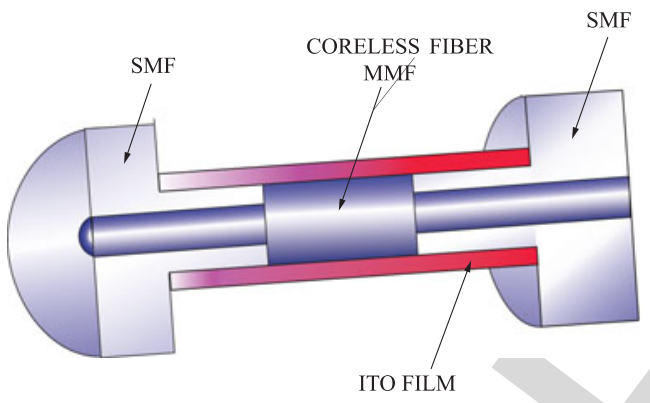

(c)

Fig. 1. (a) 14-mm segment of coreless MMF spliced on each end to standard SMF forming an SMS structure, (b) SMS structure after the HF etching, (c) etched SMS structure with an ITO thin-film deposition.

tegrated with FIMMWAVE. Finite difference method FDM was used for the SMF and MMF sections, since it is the most accurate method available for cylindrical waveguides. In the SMF sections only the fundamental mode was analyzed, whereas for the MMF section 30 modes were analyzed, thus allowing to achieve convergence in the results.

\section{A. Diameter Reduction}

Light from a white SLED source was launched through the SMS structure during the etching stage in a $40 \% \mathrm{HF}$ solution until the diameter of the fiber was reduced to approximately $25 \mu \mathrm{m}$. The transmission spectrum was recorded by an Optical Spectrum Analyzer (OSA). Fig. 2 depicts the experimental setup.

\section{B. Film Deposition}

Three SMS structures were etched with the aforementioned procedure, hereafter called Sensor 1, Sensor 2, and Sensor 3,

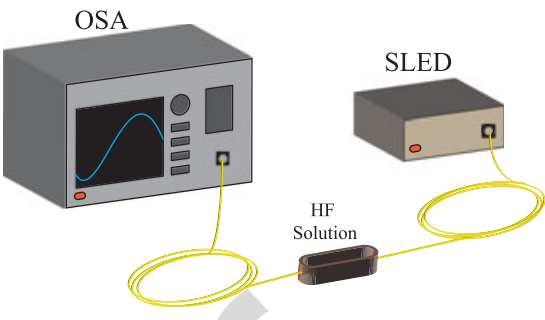

Fig. 2. Experimental setup for etching the SMS structure.

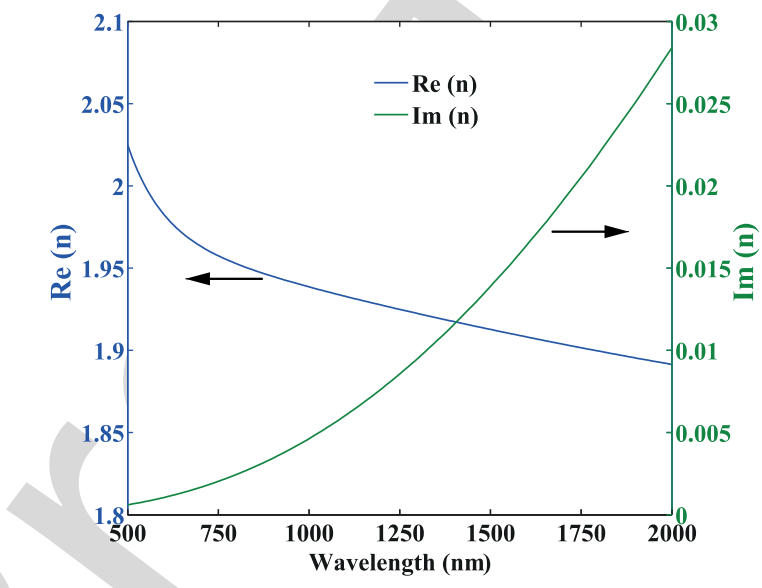

Fig. 3. Ellipsometry analysis of the ITO film used in this work.

respectively. Light from a white SLED source was launched into the structures while an ITO thin-film was deposited by sputtering (first on Sensor 2, and then on Sensor 3). An Optical Spectrum Analyzer (OSA) recorded the transmission spectra during the deposition.

Sensor 1 had no thin-film, Sensor 2 and Sensor 3 were deposited with ITO during 45 and 75 seconds respectively in a sputtering device (K675XD from Quorum Technologies, Ltd.) using $150 \mathrm{~mA}$ current and $8 \times 10^{-3} \mathrm{mbar}$ pressure.

To study the effect of the ITO thin-film deposition theoretically, it was necessary to obtain its dispersion curves. Fig. 3 depicts the ellipsometry analysis performed. This ellipsometric information allowed to compare the theoretical wavelength spectra before and after the deposition.

\section{Device Characterization by Wavelength Shift}

After the fabrication of the sensors, the same setup shown in Fig. 2 was used to characterize them when subjected to changes in the external RI. In order to observe the wavelength shift, the sensitive structure was immersed in various solutions of glycerol in water at different concentrations [13], [14]. The sensitivity curves were studied by tracking the spectral changes of the nearest attenuation band to a wavelength of $1550 \mathrm{~nm}$.

This characterization was performed to all three sensors fabricated: Sensor 1, Sensor 2, and Sensor 3.

Theoretically, the sensor characterization was performed by simulating with FIMMWAVE the SMS structure after the etching procedure for the three film conditions proposed, for different refractive indices of the surrounding medium. The refractive 


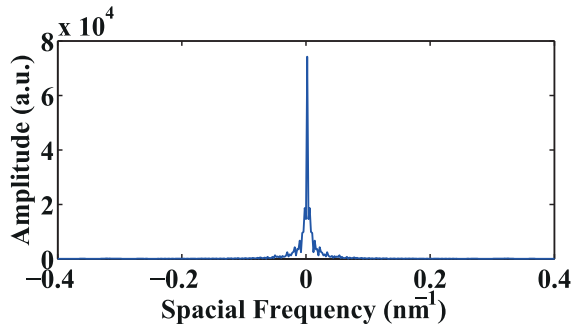

(a)

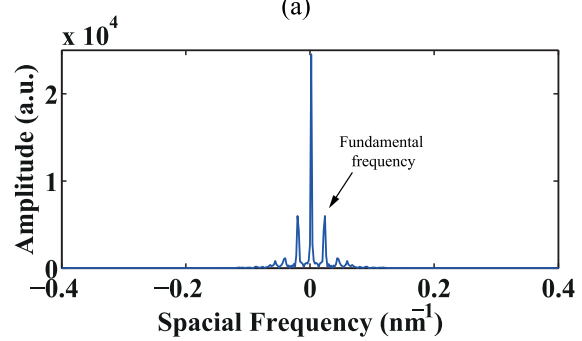

(b)

Fig. 4. Amplitude of the fast Fourier transform before (a) and after (b) of the theoretical spectrum obtained by etching.

index of the optical fiber cladding, made of fused silica, was estimated by using the Sellmeier equation:

$$
n^{2}(\omega)=1+\sum_{j=1}^{m} \frac{B_{j} \omega_{j}^{2}}{\omega_{j}^{2}-\omega^{2}}
$$

with parameters: $\mathrm{B} 1=0.691663, \mathrm{~B} 2=0.4079426, \quad \mathrm{~B} 3=$ $0.8974794, \lambda 1=0.0684043 \mu \mathrm{m}, \lambda 2=0.1162414$, and $\lambda 3=$ 9.896161, where $\lambda \mathrm{j}=2 \pi \mathrm{c} / \omega \mathrm{j}$ and $\mathrm{c}$ is the speed of light in vacuum [15]. The optical fiber core refractive index for the simulations was obtained, according to the specifications from the fiber manufacturer, by increasing the refractive index of the cladding $0.36 \%$.

\section{Degrees Brix $\left({ }^{\circ} \mathrm{Bx}\right)$ Sensor Monitored by Both Wavelength} and Phase Shift Detection

Typically, sensors are characterized by tracking the wavelength of an attenuation band using an optical spectrum analyzer, or alternatively by measuring the intensity variations at a fixed wavelength using a power meter. The fast Fourier transform (FFT) analysis, which permits to extract the phase of the optical spectrum, is not a broadly used technique despite it provides useful and clear information to be used in sensing applications and permits to use interrogators instead of optical spectrum analyzers [16], [17].

The sinusoidal spectrum of the SMS sensors after etching permits to see a sharp peak corresponding to the fundamental frequency (see in Fig. 4 the comparison between the magnitude of the fast Fourier transform before and after etching). Consequently, it is possible to obtain a phase sensitive device by tracking the phase of this fundamental frequency as a function of the parameter to detect.

In order to probe the feasibility of this method for the developed sensor shown here, a degrees Brix sensor by phase shift detection is presented. The ITO thickness film to the sensor used in this application is approximately $60 \mathrm{~nm}$. A MATLAB

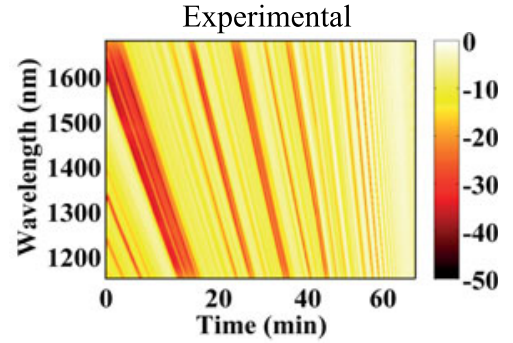

(a)

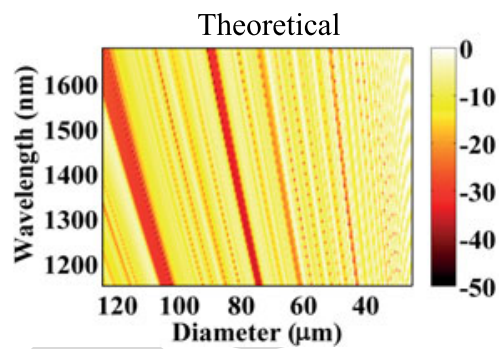

(b)

Fig. 5. Evolution of the wavelength spectrum due to reduction of the diameter by HF etching: (a) Experimental; (b) theoretical.

script was implemented to obtain the phase of the fundamental 152 frequency in the optical spectrum response of the sensor. 153

Degrees Brix is a scale of relative density used in the sugar 154 and winemaking industry. It indicates the percentage of cane 155 sugar by weight in a solution or juice of unfermented grapes. 156 Its measurement is crucial in many applications, such as fruit 157 juice, carbonated beverage industry, and wine making. $\quad 158$

The solutions used here were prepared by dissolving sucrose 159 in distilled water. One (1) ${ }^{\circ} \mathrm{Bx}$ equals one (1) gram of sucrose 160 dissolved in 100 grams of solution.

\section{E. Phase Shift and Temperature Cross Sensitivity}

A 40-nm thickness ITO thin-film deposited sensor fabricated following the same procedure described for Sensor 2 was placed in a water cell with temperature control. The temperature was set to $40{ }^{\circ} \mathrm{C}$ and the spectra started being recorded after reaching this set point, for 10 minutes. After this time, the control was set to $30{ }^{\circ} \mathrm{C}$ and kept at this constant temperature for 20 minutes. Finally, the temperature control was set back at $40{ }^{\circ} \mathrm{C}$ for 10 minutes once this temperature was reached. The real time phase shift was recorded and processed along with the entire procedure of temperature variation.

\section{RESULTS}

\section{A. Diameter Reduction}

The experimental and theoretical evolution of the wavelength spectrum as a function of the fiber diameter are depicted in Fig. 5(a) and (b) respectively.

Fig. 6(a) and (b) show the theoretical and experimental transmission spectra for both, etched and unetched fibers. Video files of this experimental and theoretical evolution can be found in the supplementary material of this manuscript. According to [9], [18], the diameter reduction is proportional to the sensitivity increase to refractive index. Consequently, a reduction from 125 to $25 \mu \mathrm{m}$ should lead to a fivefold increase. 


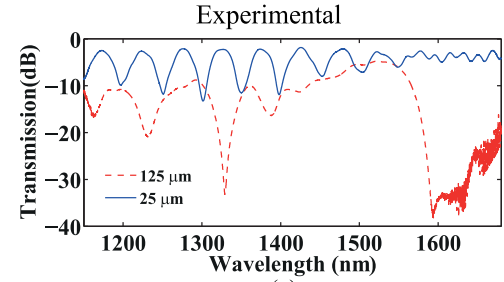

(a)

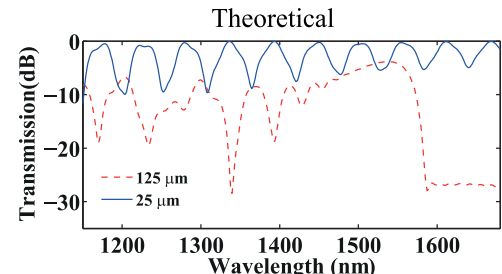

(b)

Fig. 6. Comparison between the spectra of unetched and etched fibers: (a) Experimental; (b) theoretical.

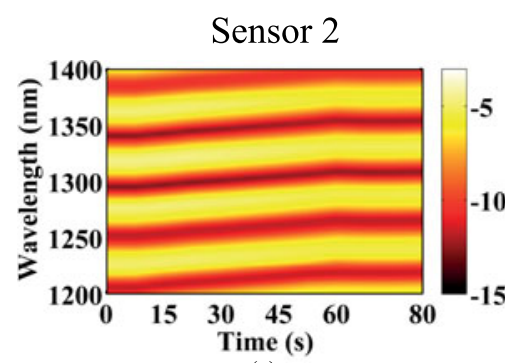

(a)

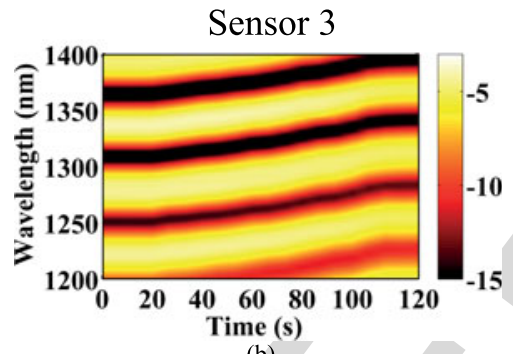

(b)

Fig. 7. Experimental evolution of the spectra during ITO deposition from: (a) Sensor 2; (b) Sensor 3.

In addition to the structural integrity of the fiber, it was observed that at this diameter the transmission evolved into a quasisinusoidal spectrum for a length of the coreless MMF of $14 \mathrm{~mm}$. This behavior facilitates both the presence of multiple attenuation bands and the shift phase study.

\section{B. Film Deposition}

Fig. 7(a) and (b) present the spectral response obtained from Sensors 2 and 3 during the ITO films deposition.

For both cases, the transmission spectra underwent redshifts during the deposition, proving the existence of a relationship between the spectral position and the ITO film thickness. Sensor 2 and Sensor 3 exhibited $23 \mathrm{~nm}$ and $40 \mathrm{~nm}$ redshifts, respectively. Fig. 8(a) and (b), and Fig. 9(a) and (b) show the experimental and theoretical spectra before and after the ITO film deposition for Sensors 2 and 3, respectively.

It can be observed that the experimental redshifts due to the ITO deposition were in essence the same as those obtained theo-
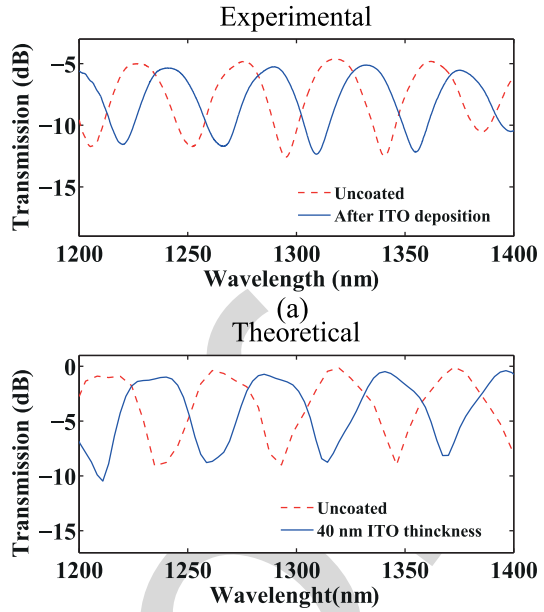

(b)

Fig. 8. Initial and final transmission spectra due to the ITO thin-film deposition on Sensor 2: (a) Experimental; (b) theoretical.
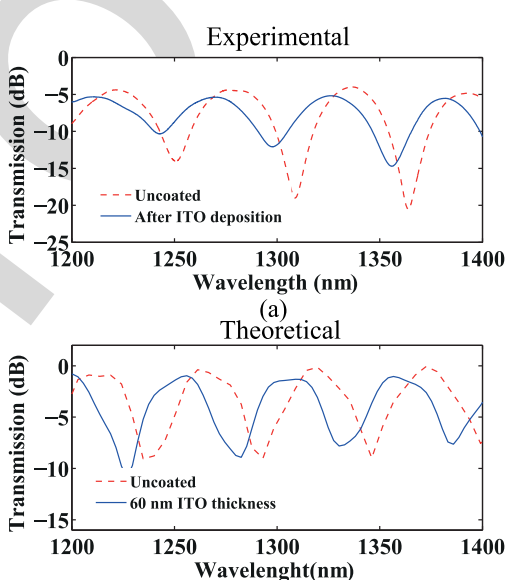

(b)

Fig. 9. Initial and final transmission spectra due to the ITO thin film deposition on Sensor 3: (a) Experimental; (b) theoretical.

retically. This probes that the transmission spectrum experiences a redshift as thin-film thickness grows.

Based on theoretical analysis, it can be concluded that the thicknesses of the deposited thin-film were of $40 \mathrm{~nm}$ and 60 $\mathrm{nm}$ to Sensor 2 and Sensor 3, respectively. To support the good match between the experimental and theoretical results, Sensor 2 was cleaved and observed using a scanning electron microscope (SEM). Fig. 10 shows the cross section, where the measurement of the film thickness was $44.18 \mathrm{~nm}$. This supposes a $10 \%$ deviation with respect to the theoretical value for this sensor.

\section{Wavelength Shift Characterization of the Device}

Figs. 11, 12, and 13 show the theoretical and experimental 213 transmission spectra as a function of wavelength for various 214 refractive indices. A redshift can be observed in all cases when 215 the RI increases, and there is a good agreement between the 216 theoretical and experimental results.

Fig. 14 illustrates the wavelength shift as a function of the 218 external refractive index, which allows the sensitivity to be cal- 219 culated both experimentally [see Fig. 14(a)] and theoretically 220 [see Fig. 14(b)]. In both cases the wavelength position was 221 taken with the attenuation band closer to $1550 \mathrm{~nm}$.

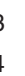

\section{.}

17

8
221 

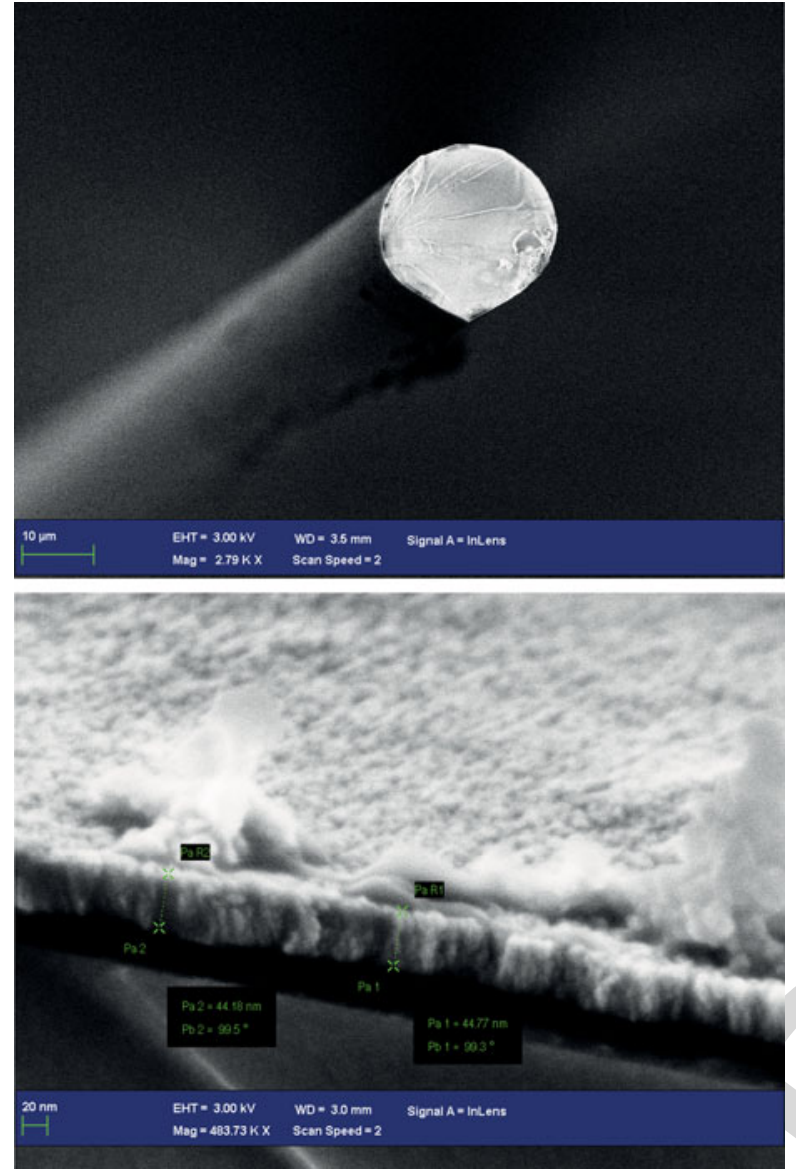

Fig. 10. SEM images of Sensor 2: Diameter $25 \mu \mathrm{m}$ and ITO thin film $44 \mathrm{~nm}$.

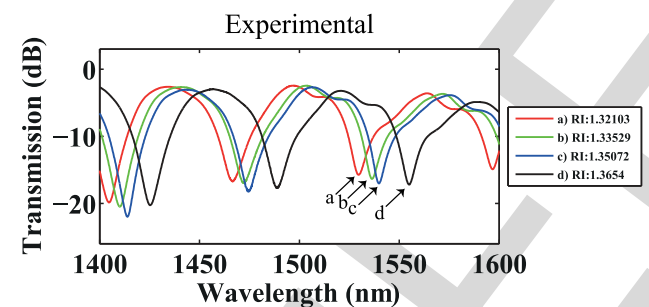

(a)

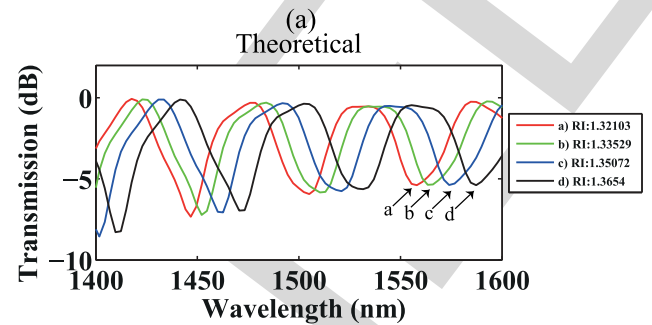

(b)

Fig. 11. Transmission spectrum for Sensor 1 as a function of the wavelength for different surrounding media refractive indices: (a) Experimental; (b) theoretical.

It can be noticed that an increase in the thin-film thickness

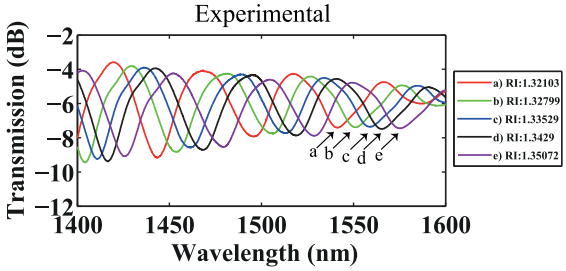

(a)

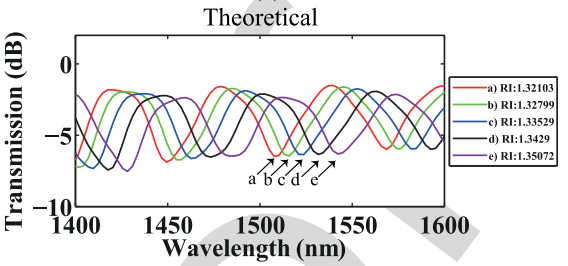

(b)

Fig. 12. Transmission spectrum for Sensor 2 as a function of the wavelength for different surrounding media refractive indices: a) Experimental; b) theoretical.

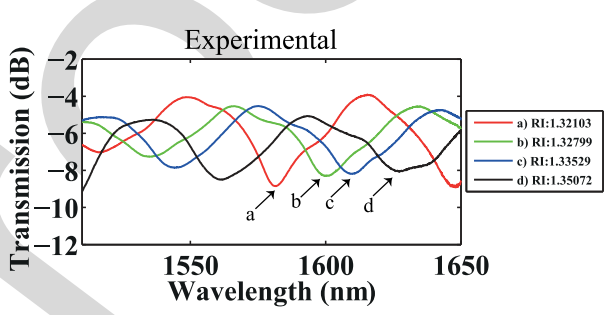

(a)

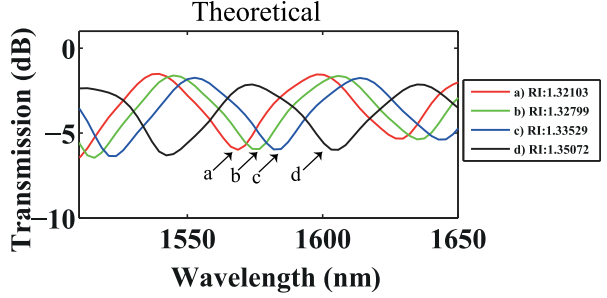

(b)

Fig. 13. Transmission a) experimental and b) theoretical for Sensor 3 as a function of the wavelength for different surrounding media refractive indices.

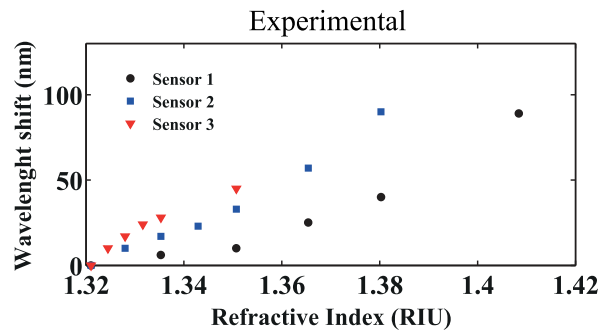

(a)

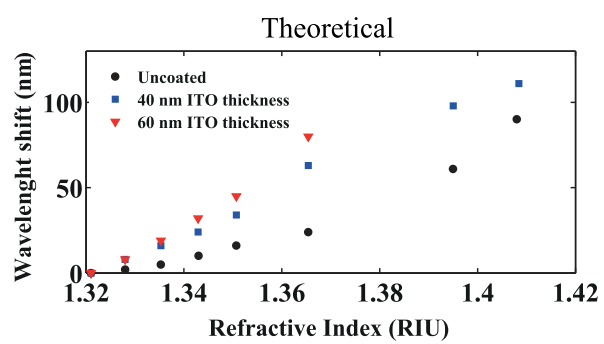

(b)

Fig. 14. Wavelength shift with refractive index to Sensor 1, 2 and 3, for both a) experimental and b) theoretical cases. 
TABLE I

COMPARISON SENSITIVITIES OBTAIN EXPERIMENTALLY AND THEORETICALLY TO A 1.32-1.35 RIU RANGE

\begin{tabular}{ccc}
\hline \hline $\begin{array}{l}\text { Thin-film } \\
\text { Thickness (nm) }\end{array}$ & Experimental Sensitivity & Theoretical Sensitivity \\
\hline \multicolumn{1}{c}{ Uncoated } & $335 \mathrm{~nm} / \mathrm{RIU}$ & $454 \mathrm{~nm} / \mathrm{RIU}$ \\
& $\mathrm{R}^{2}=0.9812$ & $\mathrm{R}^{2}=0.9669$ \\
& $1062 \mathrm{~nm} / \mathrm{RIU}$ & $1131 \mathrm{~nm} / \mathrm{RIU}$ \\
$\sim 40$ & $\mathrm{R}^{2}=0.9906$ & $\mathrm{R}^{2}=0.999$ \\
& $1442 \mathrm{~nm} / \mathrm{RIU}$ & $1536 \mathrm{~nm} / \mathrm{RIU}$ \\
$\sim 60$ & $\mathrm{R}^{2}=0.953$ & $\mathrm{R}^{2}=0.9951$ \\
\hline \hline
\end{tabular}

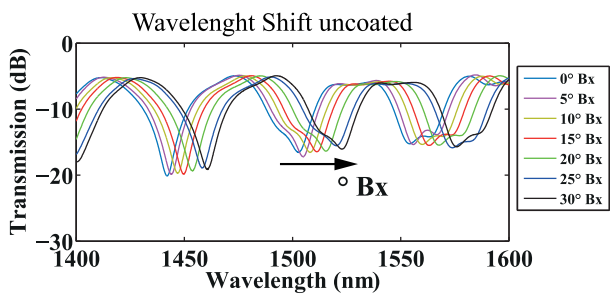

(a)

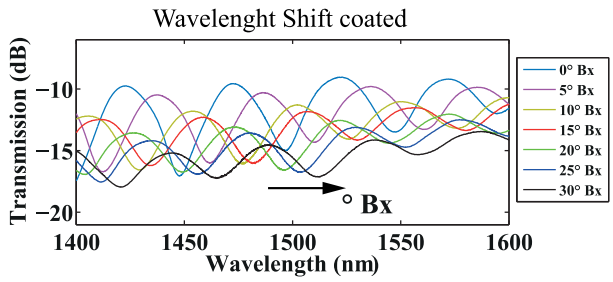

(b)

Fig. 15. Transmission as a function of the wavelength to different ${ }^{\circ} \mathrm{Bx}$ of an (a) etched SMS configuration without thin-film and (b) a $60 \mathrm{~nm}$ thin-film Thickness ITO etched SMS configuration. over, though overcome by LPFGs optimized with a hard etching [19], the device presented here is comparable with the sensitivity obtained with LPFGs optimized with a soft etching [20], which along with the possibility to monitor the phase shift indicates that it is an interesting device for biosensing applications, where a high degree of accuracy.

\section{Degrees Brix $\left({ }^{\circ} \mathrm{Bx}\right)$ Sensor Monitored by Both Wavelength and Phase Shift Detection}

Fig. 15 shows transmission as a function of the wavelength for different solutions of sucrose in water addressing two cases: an etched SMS configuration without thin-film and a $60 \mathrm{~nm}$ thickness ITO thin-film deposited on it. Both cases show a redshift when the probe was immersed in.

The magnitude of the wavelength shift is more notorious in the coated fiber, regardless of the attenuation observed, according to what has been observed in the previous section.

Fig. 16(a) and (b) present the wavelength shift of an attenuation band and the phase shift for both the etched SMS configuration without ITO coating and the same configuration type with a $60 \mathrm{~nm}$ thickness ITO thin-film. A simple linear fit of the results evidences that the ITO film permitted to obtain a three-fold sensitivity increase in both cases.

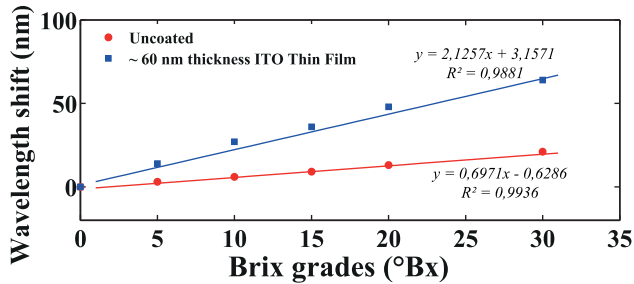

(a)

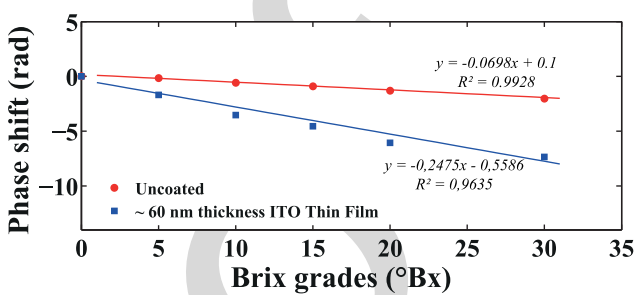

(b)

Fig. 16. Phase shift of a brix grades sensor: (a) Without ITO thin-film; (b) with $60 \mathrm{~nm}$ thickness ITO thin-film.

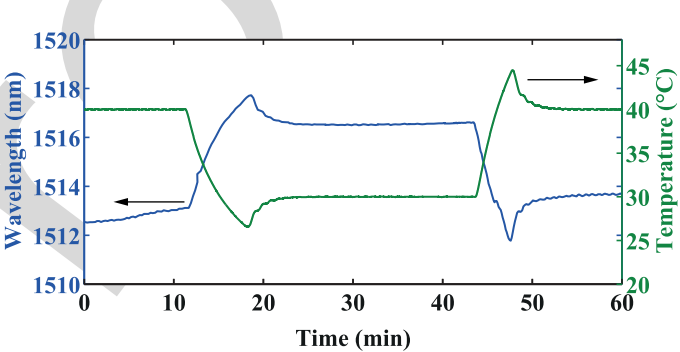

(a)

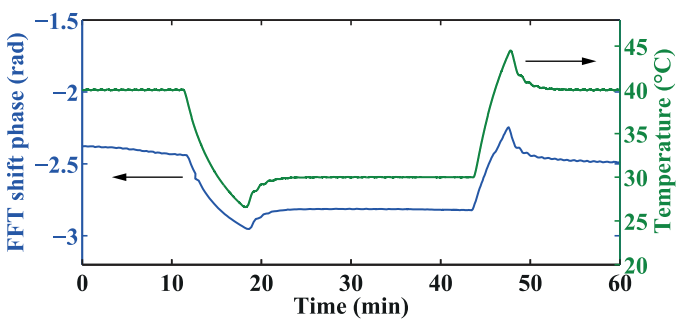

(b)

Fig. 17. (a) Wavelength shift and (b) phase shift temperature cross sensitivity.

\section{E. Temperature Cross Sensitivity}

Fig. 17(a) and (b) show the behavior of the wavelength shift 252 of an attenuation band and the phase shift with the temperature 253 setting as described in Section II-E. This probe was realized 254 with the $60 \mathrm{~nm}$ thin-film thickness sensor. The phase behavior 255 observed followed a trend imposed by the temperature's profile 256 generated as a consequence of the set points established. The 257 sensitivity was $0.3 \mathrm{~nm} /{ }^{\circ} \mathrm{C}$ and $0.034 \mathrm{rad} /{ }^{\circ} \mathrm{C}$.

A $0.14{ }^{\circ} \mathrm{Bx} /{ }^{\circ} \mathrm{C}$ of sensitivity was calculated for the ITO thinfilm coated sensors analyzed in the previous section.

\section{CONCLUSION}

This manuscript presented the optimization of SMS fiber 262 structures with a combined application of two techniques: etch- 263 ing and deposition of a thin-film. By an adequate design it 264 was possible to track both wavelength shifts of the optical spec- 265 trum and, by applying a simple FFT measurement technique, the 266 
phase shift of the fundamental frequency. The FFT measurement used in the analysis is an easy method that can be most applicable to networks that require narrow band, multiplexing capability and that have some problems related with high losses and noise.

The results also showed that the sensitivity obtained for this configuration of SMS was enhanced by reduction of the fiber diameter and by increasing the ITO film thickness. A good agreement was achieved between the experimental and the simulated approaches for this sensing device. A sensitivity of 1442 $\mathrm{nm} / \mathrm{RIU}$ was obtained by tracking the wavelength shift in a SMS with $25 \mu \mathrm{m}$ diameter and a $60 \mathrm{~nm}$ ITO thickness film, whereas for the same device, the FFT phase shift analysis showed a $0.24 \mathrm{rad} /$ RIU sensitivity.

These sensitivities, which are in the order of magnitude of other structures such as long period fiber gratings (LPFGs) (but with an inherently simpler manufacture process), place the developed sensing device as a good option for applications where high sensitivities and compact structures are required. As an example, a degrees Brix sensor has been presented, where the deposition of an ITO thin-film enhances the sensitivity of the device by a factor of 3 .

[1] C. R. Dennison, P. M. Wild, D. R. Wilson, and M. K. Gilbart, “An in-fiber Bragg grating sensor for contact force and stress measurements in articular joints," Meas. Sci. Technol., vol. 21, no. 11, 2010, Art. no. 115803.

[2] I. Del Villar, I. R. Matias, F. J. Arregui, and M. Achaerandio, "Nanodeposition of materials with complex refractive index in long-period fiber gratings," J. Lightw. Technol., vol. 23, no. 12, pp. 4192-4199, Dec. 2005.

[3] R. Kashyap, Fiber Bragg Gratings. New York, NY, USA: Academic, 1999.

[4] F. Chiavaioli et al., "Sol-gel-based titania-silica thin film overlay for long period fiber grating-based biosensors," Anal. Chem., vol. 87, no. 24, pp. 12024-12031, 2015.

[5] I. Del Villar, C. R. Zamarreño, M. Hernaez, F. J. Arregui, and I. R. Matias, "Lossy mode resonance generation with indium-tin-oxide-coated optical fibers for sensing applications," J. Lightw. Technol., vol. 28, no. 1, pp. 111-117, Jan. 2010.

[6] J. Homola, "Surface plasmon resonance sensors for detection of chemical and biological species," Chem. Rev., vol. 108, no. 2, pp. 462-493, 2008.

[7] Y. Cardona Maya, N. Gómez Cardona, and P. I. Torres Trujillo, "Low cost heat-and-pull rig for manufacturing adiabatic optical fiber tapers," Revista Facultad de Ingenieria Universidad de Antioquia, no. 70, pp. 167-172, 2014. [Online]. Available: http://aprendeenlinea.udea.edu.co/revistas/ index.php/ingenieria/article/viewFile/14820/16007

[8] T. Zhu, D. Wu, M. Liu, and D.-W. Duan, "In-line fiber optic interferometric sensors in single-mode fibers," Sensors, vol. 12, no. 8, pp. 10430-10449, 2012.

[9] I. D. Villar, A. B. Socorro, J. M. Corres, F. J. Arregui, and I. R. Matias, "Refractometric sensors based on multimode interference in a thin-film coated single-mode-multimode-single-mode structure with reflection configuration," Appl. Opt., vol. 53, no. 18, pp. 3913-3919, 2014.

[10] Q. Wang and G. Farrell, "All-fiber multimode-interference-based refractometer sensor: Proposal and design," Opt. Lett., vol. 31, no. 3, pp. 317$319,2006$.

[11] J. Lou, L. Tong, and Z. Ye, "Modeling of silica nanowires for optical sensing," Opt. Express, vol. 13, no. 6, pp. 2135-2140, 2005.

[12] A. W. Snyder and J. Love, Optical Waveguide Theory. Berlin, Germany: Springer, 2012.

[13] P. R. Cooper, "Refractive-index measurements of liquids used in conjunction with optical fibers," Appl. Opt., vol. 22, no. 19, pp. 3070-3072, 1983.

[14] L. Hoyt, "New table of the refractive index of pure glycerol at $20 \mathrm{C}$," Ind. Eng. Chem., vol. 26, no. 3, pp. 329-332, 1934.

[15] I. Malitson, "Interspecimen comparison of the refractive index of fused silica*," J. Opt. Soc. Amer., vol. 55, no. 10, pp. 1205-1209, 1965.

[16] D. Barrera et al., "Low-loss photonic crystal fiber interferometers for sensor networks," J. Lightw. Technol., vol. 28, no. 24, pp. 3542-3547, Dec. 2010.
[17] D. Leandro, M. Bravo, A. Ortigosa, and M. Lopez-Amo, "Real-time FFT analysis for interferometric sensors multiplexing," J. Lightw. Technol., vol. 33, no. 2, pp. 354-360, Jan. 2015.

[18] P. Wang, G. Brambilla, M. Ding, Y. Semenova, Q. Wu, and G. Farrell, "High-sensitivity, evanescent field refractometric sensor based on a tapered, multimode fiber interference," Opt. Lett., vol. 36, no. 12, pp. 22332235, 2011.

[19] I. Del Villar, J. L. Cruz, A. B. Socorro, J. M. Corres, and I. R. Matias, "Sensitivity optimization with cladding-etched long period fiber gratings at the dispersion turning point," Opt. Express, vol. 24, no. 16, pp. 1768017685, 2016.

[20] F. Chiavaioli et al., "Towards sensitive label-free immunosensing by mean of turn-around point long period fiber gratings," Biosensors Bioelectron., vol. 60, pp. 305-310, 2014.

Yamile Cardona-Maya received the degree in engineering physics and the M.Sc. degree in physics from the Universidad Nacional de Colombia, Medellin, Colombia, in 2011 and 2014, respectively. She is currently working toward the Ph.D. degree in sciences-physics. In 2016, she was a visiting Ph.D. student at the Public University of Navarre, Pamplona, Spain. Her research is focused on optical fiber sensors for applications in life sciences and different industries branches.

Ignacio Del Villar received the M.S. degree in electrical and electronic engineering and the Ph.D. degree, specialty in optical fiber sensors, from the Public University of Navarra, Pamplona, Spain, in 2002 and 2006, respectively.

During 2004, he was a Visiting Scientist at the Institute d'Optique, Orsay, France, and in 2005, he was a Visiting Scientist in the Department of Applied Physics, University of Valencia, Burjassot, Spain. His research interests include optical fiber sensors and the analysis of waveguides and nanostructured materials, where he has coauthored more than 100 chapter books, journals, and conference papers. He is currently a reader at the Public University of Navarra since 2008, an Associate Editor of the Optics \& Laser Technology Journal since 2012, and an Associate Editor of the Journal of Sensors since 2014.

Abian B. Socorro received the M.Sc. degree in biomedical engineering and the Ph.D. degree in engineering both from the Public University of Navarre (PUNUPNA), Pamplona, Spain, in 2012 and 2015, respectively. Since 2010, he has been an Electrical and Electronic Engineer. In 2014, he was a visiting Ph.D. student in the Armani Research Laboratory, University of Southern California Los Angeles, CA, USA. He is currently working as an Assistant Professor at the PUN-UPNA. His research interests include fiber-optic biosensors, fiber in the body, surface biofunctionalization, and other biomedical engineering applications.

Jesus M. Corres received the M.S. degree in electrical engineering and the Ph.D degree both from the Public University of Navarra (UPNA), Pamplona, Spain, in 1996 and 2003, respectively. He is currently working as an Associate Professor in the Department of Electrical and Electronic Engineering, UPNA. He is the author or coauthor of more than 100 publications. His main research interests include the development of fiber-optic sensors using nanostructured materials for biomedical, environmental, and safety applications. He is an Associate Editor of the IEEE SENSOR LETTERS and Hindawi Journal of Sensors.

Ignacio R. Matias received the M.S. degree in electrical and electronic engineering and the Ph.D. degree in optical fiber sensors from the Polytechnic University of Madrid, Madrid, Spain, in 1992 and 1996, respectively. In 1996 , he became a Lecturer at the Public University of Navarra, Pamplona, Spain, where currently he is a permanent Professor. He has coauthored more than 300 chapter books, journals, and conference papers related to optical fiber sensors and passive optical devices and systems. He is a Senior and Topical Editor of the IEEE SENSORS JOURNAL.

Juan F. Botero-Cadavid was born in Colombia in 1979. He received the B.S. and M.S. degrees in mechanical engineering and physics, from the Universidad Nacional de Colombia, Medellin, Colombia, in 2004 and 2007, respectively, and the Ph.D. degree in mechanical engineering from the University of Victoria, Victoria, BC, Canada, in 2014.

Since 2008, he has been an Assistant Professor in the School of Physics, Universidad Nacional de Colombia. His research interests include, but are not limited to, mechanical design, optical fiber sensors, digital holography, machining, and polymer electrolyte fuel cells. 


\title{
Wavelength and Phase Detection Based SMS Fiber Sensors Optimized With Etching and Nanodeposition
}

\author{
Yamile Cardona-Maya, Ignacio Del Villar, Abian B. Socorro, Jesus M. Corres, Member, IEEE, \\ Ignacio R. Matias, Senior Member, IEEE, and Juan F. Botero-Cadavid
}

\begin{abstract}
The development of an optical fiber refractometer by hydrogen fluoride etching and sputtering deposition of a thin-film of indium tin oxide on a single-mode-multimode-single-mode fiber structure has been analyzed with the aim of improving the sensitivity to the changes of the refractive index (RI) of the external medium. The device is sensitive to the RI changes of the surrounding medium, which can be monitored by tracking the spectral changes of an attenuation band or with a fast Fourier transform (FFT) analysis. By using an optical spectrum analyzer combined with a simple FFT measurement technique, the simultaneous realtime monitoring is achieved. The results show that the sensitivity depends on the thin-film thickness. A maximum of $1442 \mathrm{~nm} / \mathrm{RIU}$ (refractive index unit) in the 1.32-1.35 RIU range has been attained. In addition, a theoretical analysis has been performed, where simulations matched with the experimental results. As a practical application of the developed optical fiber structure, $\mathrm{a}^{\circ} \mathrm{Brix}\left({ }^{\circ} \mathrm{Bx}\right)$ sensor has been implemented with a sensitivity of $2.13 \mathrm{~nm} /{ }^{\circ} \mathrm{Bx}$ and 0.25 $\mathrm{rad} /{ }^{\circ} \mathrm{Bx}$ respectively for wavelength and phase shift detection.
\end{abstract}

Index Terms-Etching, optical fiber sensor, refractive index, single-mode-multimode-single-mode (SMS), thin-films.

\section{$\mathbf{O}$} PTICAL fiber refractometers have been extensively studied for chemical, medicine, and biological applications due their multiple advantages such as compact size and high resolution. They can be used in harsh environments and allow for minimally invasive procedures to be performed [1].

Up until now, several technologies have been used to develop optical fiber refractometers. Some of these technologies include: fiber gratings [2], [3], long period fiber gratings [4], resonances [5], [6], evanescent field [7] and interferometers [8].

Manuscript received April 21, 2017; revised June 5, 2017; accepted June 16, 2017. This work was supported in part by the Agencia Estatal de Investigación, in part by Fondo Europeo de Desarrollo Regional (TEC2016-78047-R), in part by the Government of Navarre through its projects with references: 2016/PI008, 2016/PC025, and 2016/PC026, and in part by the Colombian Administrative Department of Science, Technology and Innovation-Colciencias, through the Program for national doctorates, calling 617 of 2013. (Corresponding author: Yamile Cardona-Maya.)

Y. Cardona-Maya and J. F. Botero-Cadavid are with the School of Physics, Universidad Nacional de Colombia, Medellin 050034, Colombia (e-mail: ycardon@unal.edu.co; jfbotero@unal.edu.co).

I. Del Villar, A. B. Socorro, J. M. Corres, and I. R. Matias are with the Department of Electrical and Electronic Engineering, Institute of Smart Cities, Public University of Navarre, Pamplona 31006, Spain (e-mail: ignacio.delvillar@unavarra.es; ab.socorro@unavarra.es; jmcorres@unavarra. es; natxo@unavarra.es).

Color versions of one or more of the figures in this paper are available online at http://ieeexplore.ieee.org.

Digital Object Identifier 10.1109/JLT.2017.2719923
Within the last group, a single-mode-multimode-single-mode SMS fiber refractometer has been studied and a sensitivity of $1199.8 \mathrm{~nm} /$ RIU for the range of refractive indices from 1.321 to 1.382 has been reported [9]. In this work, the experimental and theoretical demonstration of a novel and high-sensitivity refractometric sensor is reported. The developed sensor relies on multimodal interference in etched SMS fiber structures coated with a thin-film that improves the previously sensitivity reported with this structure [9]. Moreover, by application of fast Fourier transform analysis (FFT), it is possible to use both wavelength and phase monitoring of the parameter to detect.

The proposed SMS configuration of this work consists of input and output single-mode fibers (SMFs) that are spliced to a section of a multimode coreless fiber (MMF) of a certain length. The operation mechanism of this refractometer is based on the multimode interference (MMI). When the light propagating along the input SMF enters the MMF section, several eigenmodes of the MMF are excited and interference among different modes occurs during the propagation along the MMF section. Finally, the light is coupled into the output SMF at the end of the MMF section [10]. Through the reduction of the diameter in the MMF section, the evanescent field penetrates further into the surrounding medium, thus increasing the sensitivity [11], [12]. Furthermore, a deposited high refractive index thin-film enhances the interaction with the environment surrounding the fiber and also permits to increase the sensitivity.

To sum up, the enhancement of the sensitivity in the SMS configuration in this work is achieved by gathering two different phenomena, diameter reduction and thin-film deposition.

\section{Methods AND MAterials}

Coreless MMF segments from POFC Inc. (Taiwan) and standard SMF pigtails from Telnet Redes Inteligentes Inc. (Zaragoza, Spain) were used in this study. The SMS structure consists on a 14-mm segment of coreless MMF spliced on each end to standard SMF pigtails, as shown in Fig. 1(a). This structure was etched using hydrofluoric acid $40 \%$ (HF) until the diameter of the fiber was reduced to approximately $25 \mu \mathrm{m}$ [see Fig. 1(b)]. This process took 70 minutes. Finally, a thin-film of Indium Tin Oxide (ITO) was deposited by sputtering on the etched region [see Fig. 1(c)]. The effect of the etching and the deposition was studied theoretically and experimentally.

The theoretical analysis was performed with FIMMWAVE. The propagation was obtained with FIMMPROP, a module in- 


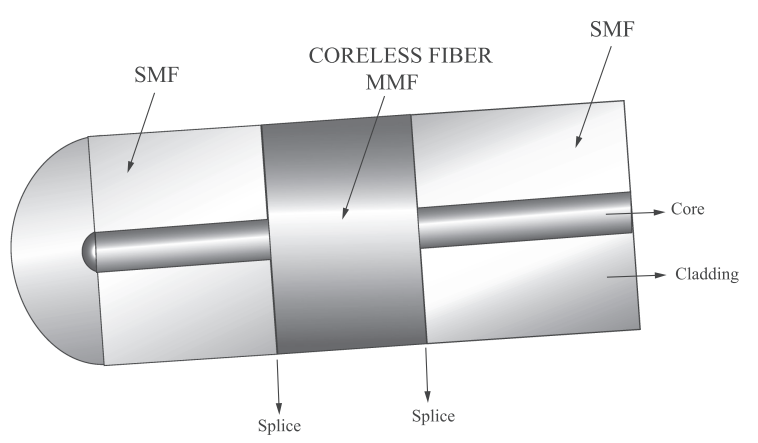

(a)

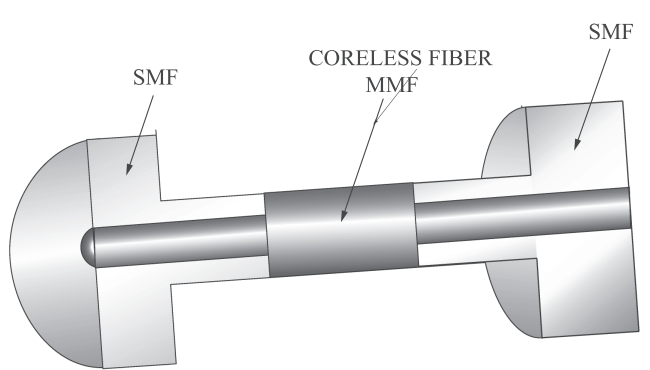

(b)

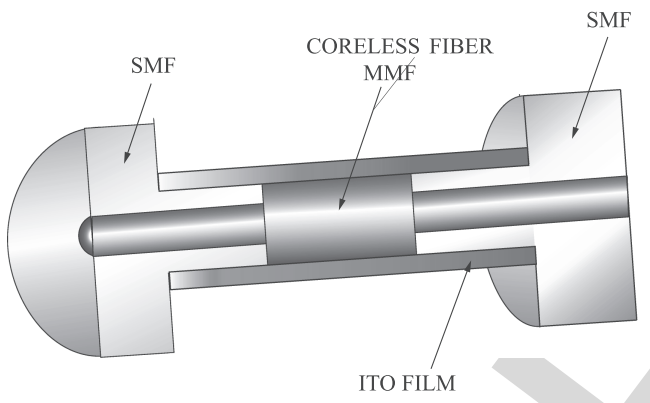

(c)

Fig. 1. (a) 14-mm segment of coreless MMF spliced on each end to standard SMF forming an SMS structure, (b) SMS structure after the HF etching, (c) etched SMS structure with an ITO thin-film deposition.

tegrated with FIMMWAVE. Finite difference method FDM was used for the SMF and MMF sections, since it is the most accurate method available for cylindrical waveguides. In the SMF sections only the fundamental mode was analyzed, whereas for the MMF section 30 modes were analyzed, thus allowing to achieve convergence in the results.

\section{A. Diameter Reduction}

Light from a white SLED source was launched through the SMS structure during the etching stage in a $40 \% \mathrm{HF}$ solution until the diameter of the fiber was reduced to approximately $25 \mu \mathrm{m}$. The transmission spectrum was recorded by an Optical Spectrum Analyzer (OSA). Fig. 2 depicts the experimental setup.

\section{B. Film Deposition}

Three SMS structures were etched with the aforementioned procedure, hereafter called Sensor 1, Sensor 2, and Sensor 3,

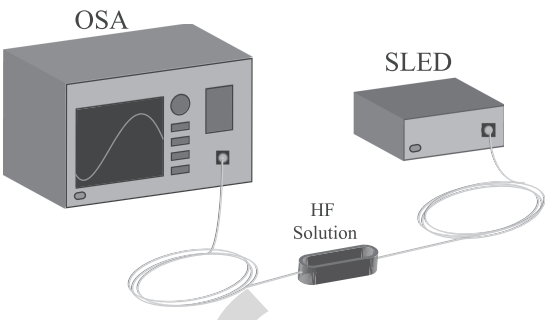

Fig. 2. Experimental setup for etching the SMS structure.

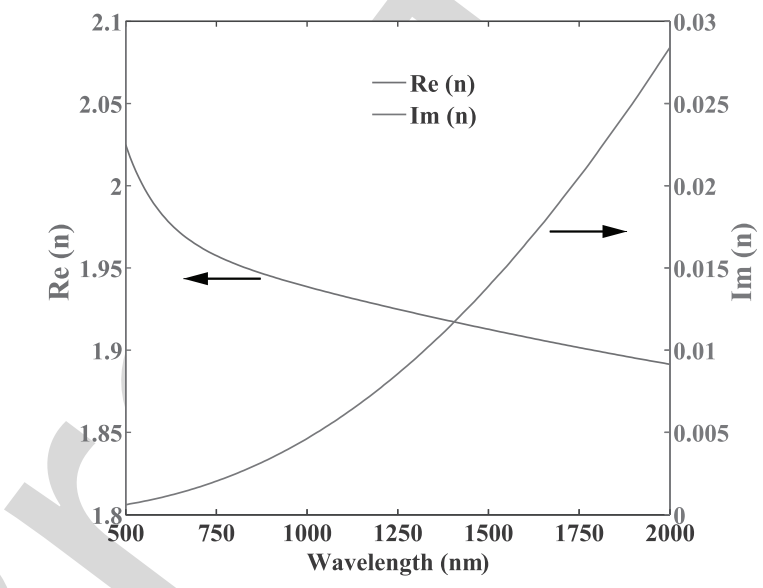

Fig. 3. Ellipsometry analysis of the ITO film used in this work.

respectively. Light from a white SLED source was launched into the structures while an ITO thin-film was deposited by sputtering (first on Sensor 2, and then on Sensor 3). An Optical Spectrum Analyzer (OSA) recorded the transmission spectra during the deposition.

Sensor 1 had no thin-film, Sensor 2 and Sensor 3 were deposited with ITO during 45 and 75 seconds respectively in a sputtering device (K675XD from Quorum Technologies, Ltd.) using $150 \mathrm{~mA}$ current and $8 \times 10^{-3} \mathrm{mbar}$ pressure.

To study the effect of the ITO thin-film deposition theoretically, it was necessary to obtain its dispersion curves. Fig. 3 depicts the ellipsometry analysis performed. This ellipsometric information allowed to compare the theoretical wavelength spectra before and after the deposition.

\section{Device Characterization by Wavelength Shift}

After the fabrication of the sensors, the same setup shown in Fig. 2 was used to characterize them when subjected to changes in the external RI. In order to observe the wavelength shift, the sensitive structure was immersed in various solutions of glycerol in water at different concentrations [13], [14]. The sensitivity curves were studied by tracking the spectral changes of the nearest attenuation band to a wavelength of $1550 \mathrm{~nm}$.

This characterization was performed to all three sensors fabricated: Sensor 1, Sensor 2, and Sensor 3.

Theoretically, the sensor characterization was performed by simulating with FIMMWAVE the SMS structure after the etching procedure for the three film conditions proposed, for different refractive indices of the surrounding medium. The refractive 


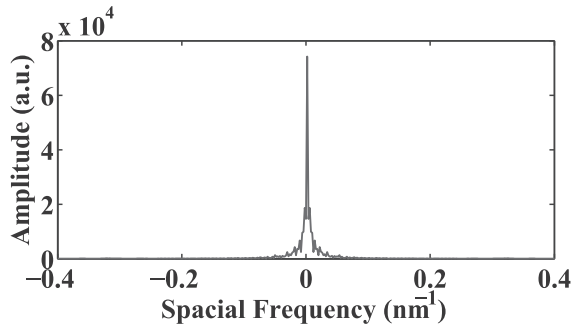

(a)

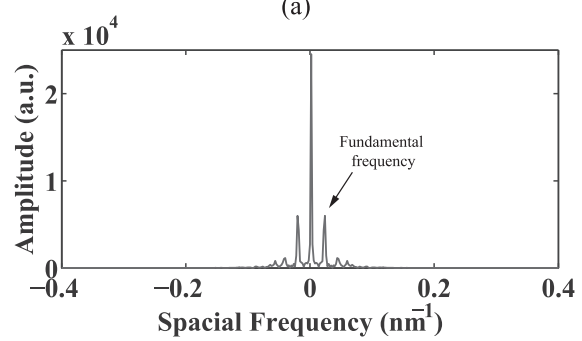

(b)

Fig. 4. Amplitude of the fast Fourier transform before (a) and after (b) of the theoretical spectrum obtained by etching.

index of the optical fiber cladding, made of fused silica, was estimated by using the Sellmeier equation:

$$
n^{2}(\omega)=1+\sum_{j=1}^{m} \frac{B_{j} \omega_{j}^{2}}{\omega_{j}^{2}-\omega^{2}}
$$

with parameters: $\mathrm{B} 1=0.691663, \mathrm{~B} 2=0.4079426, \quad \mathrm{~B} 3=$ $0.8974794, \lambda 1=0.0684043 \mu \mathrm{m}, \lambda 2=0.1162414$, and $\lambda 3=$ 9.896161, where $\lambda \mathrm{j}=2 \pi \mathrm{c} / \omega \mathrm{j}$ and $\mathrm{c}$ is the speed of light in vacuum [15]. The optical fiber core refractive index for the simulations was obtained, according to the specifications from the fiber manufacturer, by increasing the refractive index of the cladding $0.36 \%$.

\section{Degrees Brix $\left({ }^{\circ} \mathrm{Bx}\right)$ Sensor Monitored by Both Wavelength} and Phase Shift Detection

Typically, sensors are characterized by tracking the wavelength of an attenuation band using an optical spectrum analyzer, or alternatively by measuring the intensity variations at a fixed wavelength using a power meter. The fast Fourier transform (FFT) analysis, which permits to extract the phase of the optical spectrum, is not a broadly used technique despite it provides useful and clear information to be used in sensing applications and permits to use interrogators instead of optical spectrum analyzers [16], [17].

The sinusoidal spectrum of the SMS sensors after etching permits to see a sharp peak corresponding to the fundamental frequency (see in Fig. 4 the comparison between the magnitude of the fast Fourier transform before and after etching). Consequently, it is possible to obtain a phase sensitive device by tracking the phase of this fundamental frequency as a function of the parameter to detect.

In order to probe the feasibility of this method for the developed sensor shown here, a degrees Brix sensor by phase shift detection is presented. The ITO thickness film to the sensor used in this application is approximately $60 \mathrm{~nm}$. A MATLAB

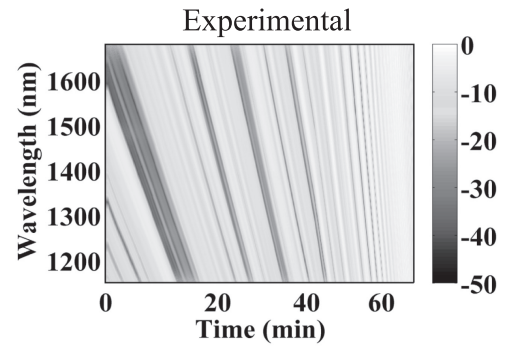

(a)

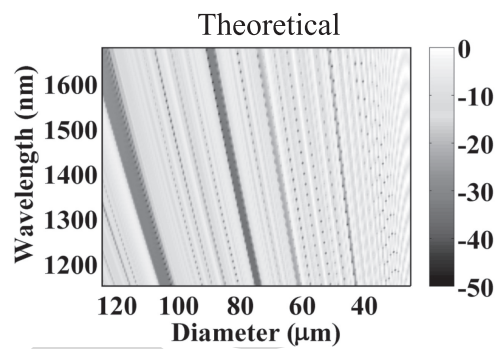

(b)

Fig. 5. Evolution of the wavelength spectrum due to reduction of the diameter by HF etching: (a) Experimental; (b) theoretical.

script was implemented to obtain the phase of the fundamental 152 frequency in the optical spectrum response of the sensor. 153

Degrees Brix is a scale of relative density used in the sugar 154 and winemaking industry. It indicates the percentage of cane 155 sugar by weight in a solution or juice of unfermented grapes. 156 Its measurement is crucial in many applications, such as fruit 157 juice, carbonated beverage industry, and wine making. $\quad 158$

The solutions used here were prepared by dissolving sucrose 159 in distilled water. One (1) ${ }^{\circ} \mathrm{Bx}$ equals one (1) gram of sucrose 160 dissolved in 100 grams of solution.

\section{E. Phase Shift and Temperature Cross Sensitivity}

A 40-nm thickness ITO thin-film deposited sensor fabricated following the same procedure described for Sensor 2 was placed in a water cell with temperature control. The temperature was set to $40{ }^{\circ} \mathrm{C}$ and the spectra started being recorded after reaching this set point, for 10 minutes. After this time, the control was set to $30{ }^{\circ} \mathrm{C}$ and kept at this constant temperature for 20 minutes. Finally, the temperature control was set back at $40^{\circ} \mathrm{C}$ for 10 minutes once this temperature was reached. The real time phase shift was recorded and processed along with the entire procedure of temperature variation.

\section{RESULTS}

\section{A. Diameter Reduction}

The experimental and theoretical evolution of the wavelength spectrum as a function of the fiber diameter are depicted in Fig. 5(a) and (b) respectively.

Fig. 6(a) and (b) show the theoretical and experimental transmission spectra for both, etched and unetched fibers. Video files of this experimental and theoretical evolution can be found in the supplementary material of this manuscript. According to [9], [18], the diameter reduction is proportional to the sensitivity increase to refractive index. Consequently, a reduction from 125 to $25 \mu \mathrm{m}$ should lead to a fivefold increase.

8

59




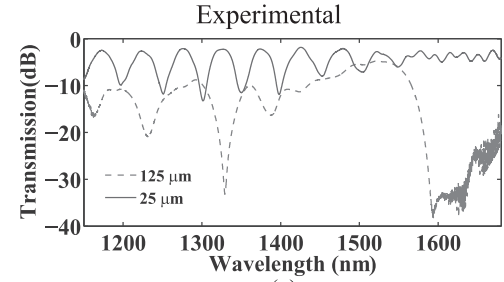

(a)

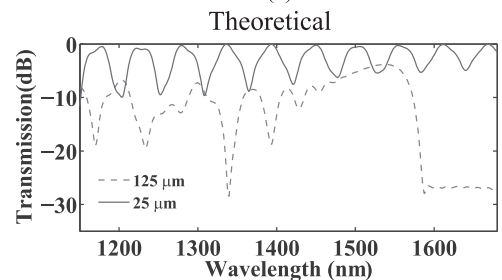

(b)

Fig. 6. Comparison between the spectra of unetched and etched fibers: (a) Experimental; (b) theoretical.

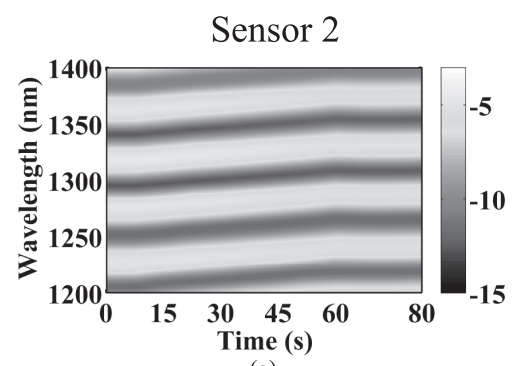

(a)

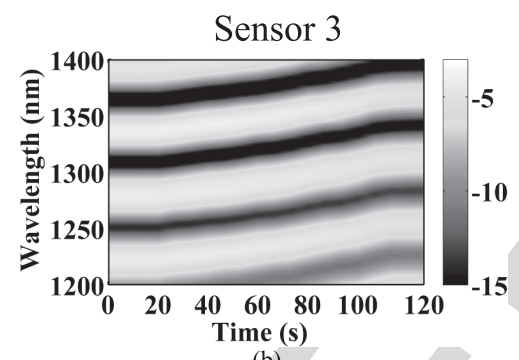

(b)

Fig. 7. Experimental evolution of the spectra during ITO deposition from: (a) Sensor 2; (b) Sensor 3 .

In addition to the structural integrity of the fiber, it was observed that at this diameter the transmission evolved into a quasisinusoidal spectrum for a length of the coreless MMF of $14 \mathrm{~mm}$. This behavior facilitates both the presence of multiple attenuation bands and the shift phase study.

\section{B. Film Deposition}

Fig. 7(a) and (b) present the spectral response obtained from Sensors 2 and 3 during the ITO films deposition.

For both cases, the transmission spectra underwent redshifts during the deposition, proving the existence of a relationship between the spectral position and the ITO film thickness. Sensor 2 and Sensor 3 exhibited $23 \mathrm{~nm}$ and $40 \mathrm{~nm}$ redshifts, respectively. Fig. 8(a) and (b), and Fig. 9(a) and (b) show the experimental and theoretical spectra before and after the ITO film deposition for Sensors 2 and 3, respectively.

It can be observed that the experimental redshifts due to the ITO deposition were in essence the same as those obtained theo-

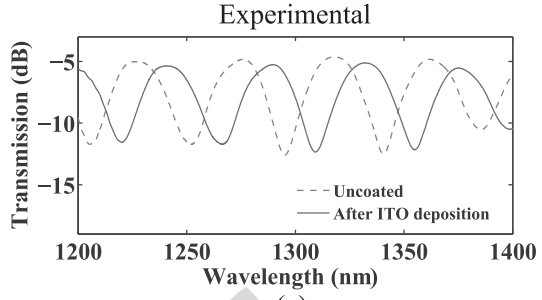

(a)

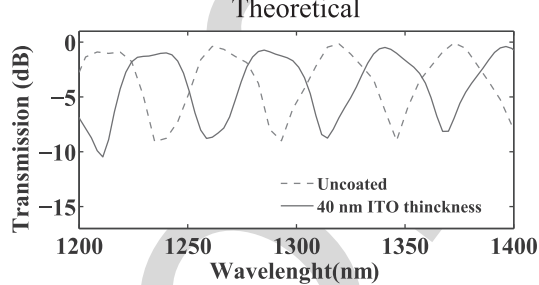

(b)

Fig. 8. Initial and final transmission spectra due to the ITO thin-film deposition on Sensor 2: (a) Experimental; (b) theoretical.
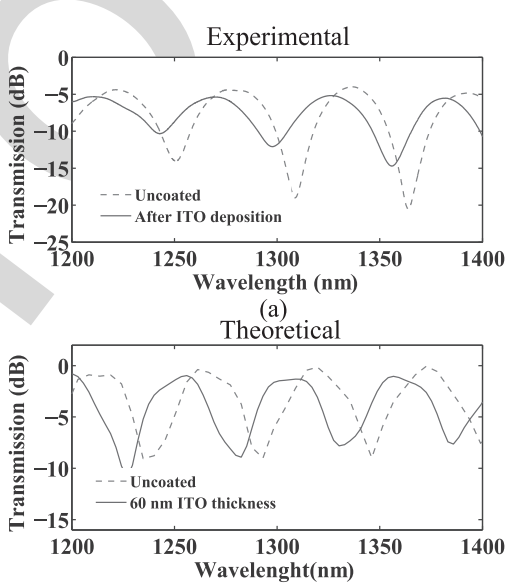

(b)

Fig. 9. Initial and final transmission spectra due to the ITO thin film deposition on Sensor 3: (a) Experimental; (b) theoretical.

retically. This probes that the transmission spectrum experiences a redshift as thin-film thickness grows.

Based on theoretical analysis, it can be concluded that the thicknesses of the deposited thin-film were of $40 \mathrm{~nm}$ and 60 $\mathrm{nm}$ to Sensor 2 and Sensor 3, respectively. To support the good match between the experimental and theoretical results, Sensor 2 was cleaved and observed using a scanning electron microscope (SEM). Fig. 10 shows the cross section, where the measurement of the film thickness was $44.18 \mathrm{~nm}$. This supposes a $10 \%$ deviation with respect to the theoretical value for this sensor.

\section{Wavelength Shift Characterization of the Device}

Figs. 11, 12, and 13 show the theoretical and experimental 213 transmission spectra as a function of wavelength for various 214 refractive indices. A redshift can be observed in all cases when 215 the RI increases, and there is a good agreement between the 216 theoretical and experimental results.

Fig. 14 illustrates the wavelength shift as a function of the 218 external refractive index, which allows the sensitivity to be cal- 219 culated both experimentally [see Fig. 14(a)] and theoretically 220 [see Fig. 14(b)]. In both cases the wavelength position was 221 taken with the attenuation band closer to $1550 \mathrm{~nm}$. . (1) 10 . (1) , 

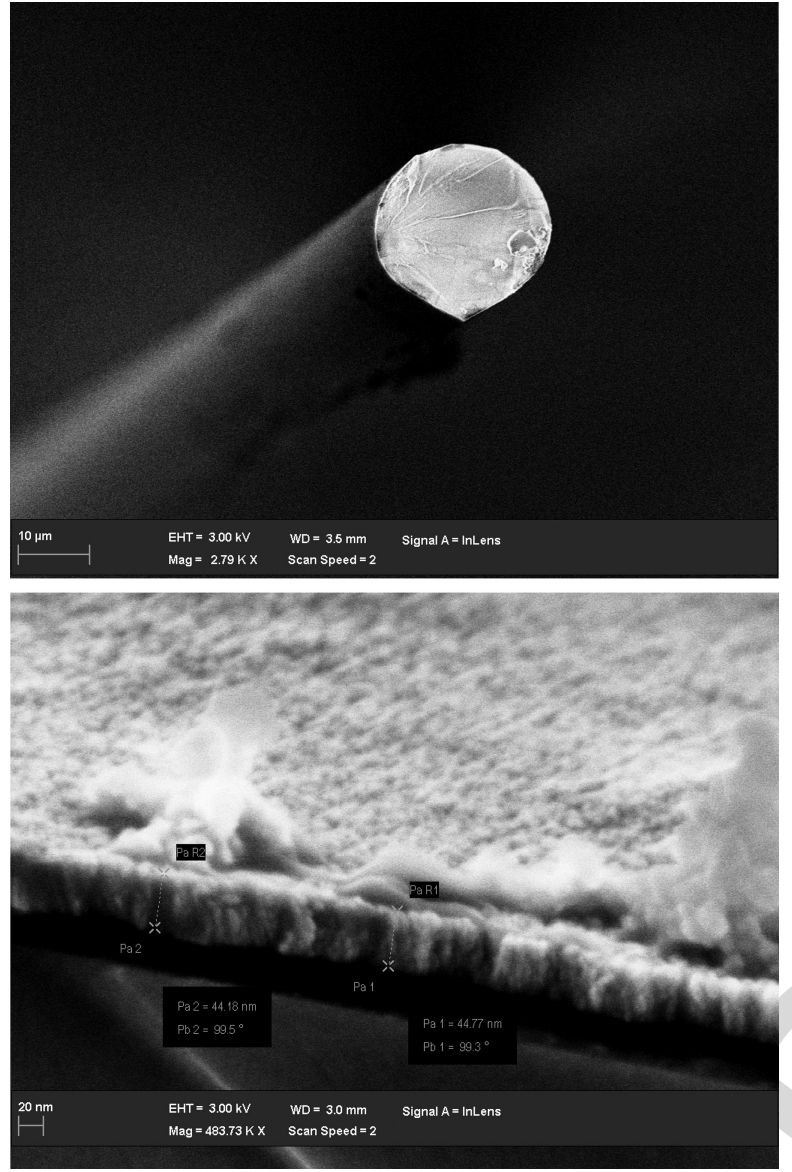

Fig. 10. SEM images of Sensor 2: Diameter $25 \mu \mathrm{m}$ and ITO thin film $44 \mathrm{~nm}$.

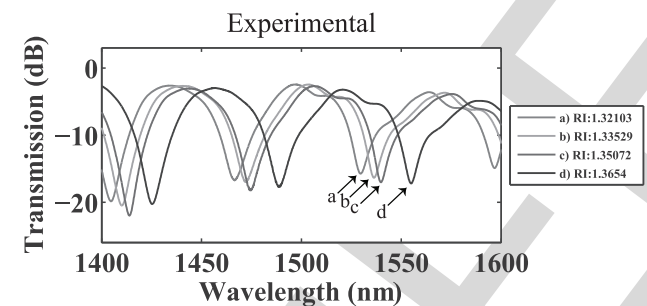

(a)

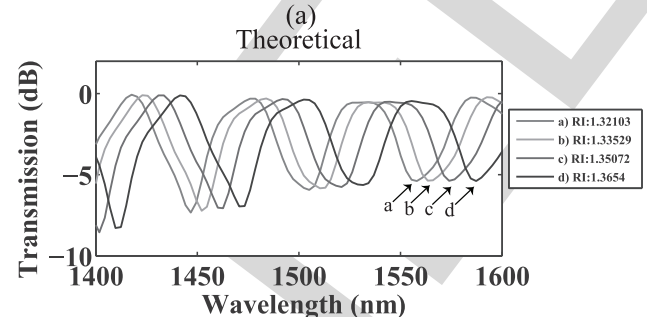

(b)

Fig. 11. Transmission spectrum for Sensor 1 as a function of the wavelength for different surrounding media refractive indices: (a) Experimental; (b) theoretical.

It can be noticed that an increase in the thin-film thickness leads to a higher sensitivity. Table I shows a summary of the sensitivities in the 1.32-1.35 refractive index range. The experimental sensitivity improves that obtained in [9]. Indeed, even though a sensitivity of $1200 \mathrm{~nm} / \mathrm{RIU}$ was attained in that work, the SRI range was $1.32-1.38$, with a higher sensitivity. More-

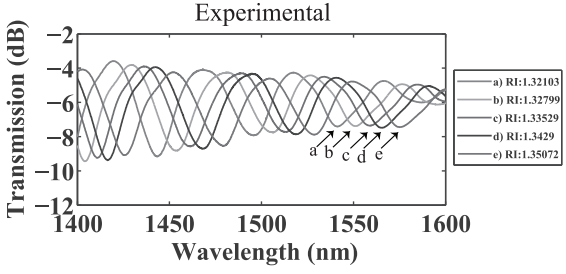

(a)

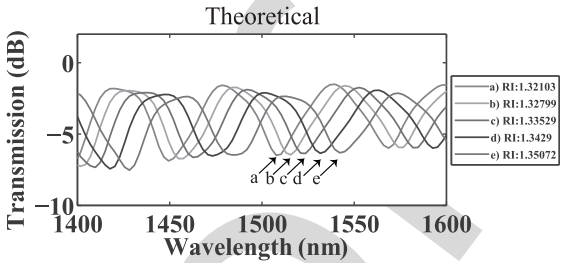

(b)

Fig. 12. Transmission spectrum for Sensor 2 as a function of the wavelength for different surrounding media refractive indices: a) Experimental; b) theoretical.

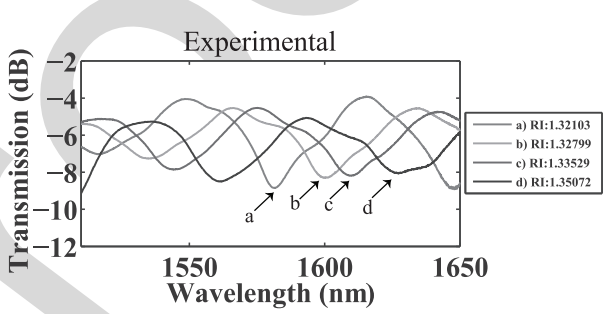

(a)

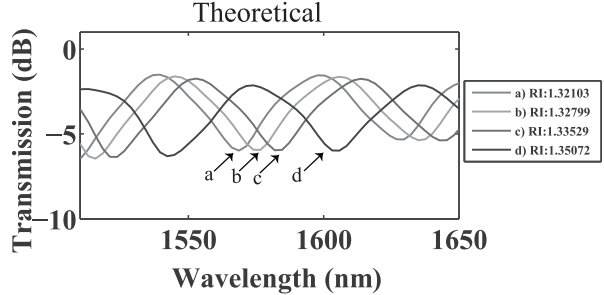

(b)

Fig. 13. Transmission a) experimental and b) theoretical for Sensor 3 as a function of the wavelength for different surrounding media refractive indices.

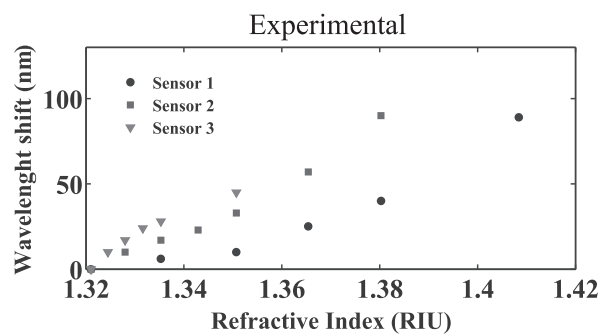

(a)

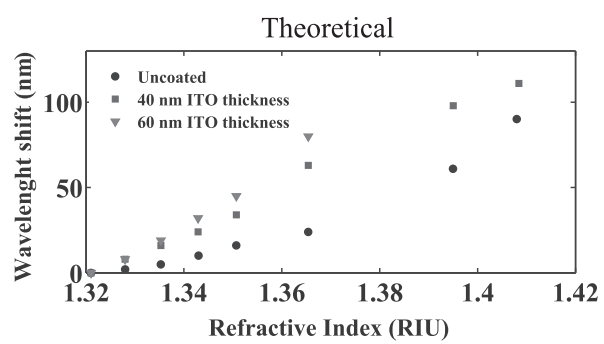

(b)

Fig. 14. Wavelength shift with refractive index to Sensor 1, 2 and 3, for both a) experimental and b) theoretical cases. 
TABLE I

COMPARISON SENSITIVITIES OBTAIN EXPERIMENTALLY AND THEORETICALLY TO A 1.32-1.35 RIU RANGE

\begin{tabular}{ccc}
\hline \hline $\begin{array}{l}\text { Thin-film } \\
\text { Thickness (nm) }\end{array}$ & Experimental Sensitivity & Theoretical Sensitivity \\
\hline \multicolumn{1}{c}{ Uncoated } & $335 \mathrm{~nm} / \mathrm{RIU}$ & $454 \mathrm{~nm} / \mathrm{RIU}$ \\
& $\mathrm{R}^{2}=0.9812$ & $\mathrm{R}^{2}=0.9669$ \\
& $1062 \mathrm{~nm} / \mathrm{RIU}$ & $1131 \mathrm{~nm} / \mathrm{RIU}$ \\
$\sim 40$ & $\mathrm{R}^{2}=0.9906$ & $\mathrm{R}^{2}=0.999$ \\
& $1442 \mathrm{~nm} / \mathrm{RIU}$ & $1536 \mathrm{~nm} / \mathrm{RIU}$ \\
$\sim 60$ & $\mathrm{R}^{2}=0.953$ & $\mathrm{R}^{2}=0.9951$ \\
\hline \hline
\end{tabular}

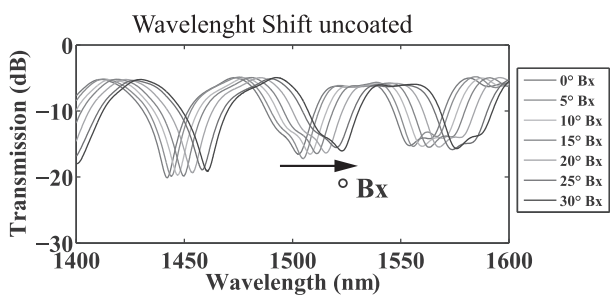

(a)

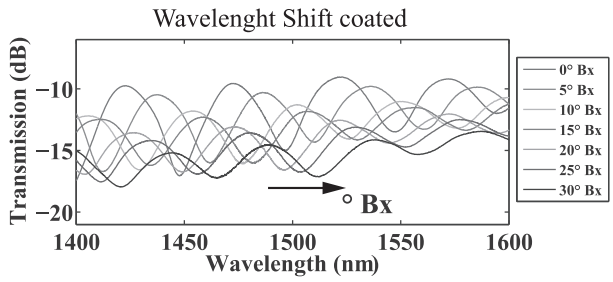

(b)

Fig. 15. Transmission as a function of the wavelength to different ${ }^{\circ} \mathrm{Bx}$ of an (a) etched SMS configuration without thin-film and (b) a $60 \mathrm{~nm}$ thin-film Thickness ITO etched SMS configuration. over, though overcome by LPFGs optimized with a hard etching [19], the device presented here is comparable with the sensitivity obtained with LPFGs optimized with a soft etching [20], which along with the possibility to monitor the phase shift indicates that it is an interesting device for biosensing applications, where a high degree of accuracy.

\section{Degrees Brix $\left({ }^{\circ} \mathrm{Bx}\right)$ Sensor Monitored by Both Wavelength and Phase Shift Detection}

Fig. 15 shows transmission as a function of the wavelength for different solutions of sucrose in water addressing two cases: an etched SMS configuration without thin-film and a $60 \mathrm{~nm}$ thickness ITO thin-film deposited on it. Both cases show a redshift when the probe was immersed in.

The magnitude of the wavelength shift is more notorious in the coated fiber, regardless of the attenuation observed, according to what has been observed in the previous section.

Fig. 16(a) and (b) present the wavelength shift of an attenuation band and the phase shift for both the etched SMS configuration without ITO coating and the same configuration type with a $60 \mathrm{~nm}$ thickness ITO thin-film. A simple linear fit of the results evidences that the ITO film permitted to obtain a three-fold sensitivity increase in both cases.

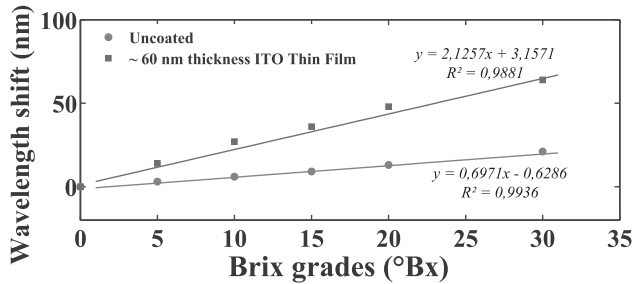

(a)

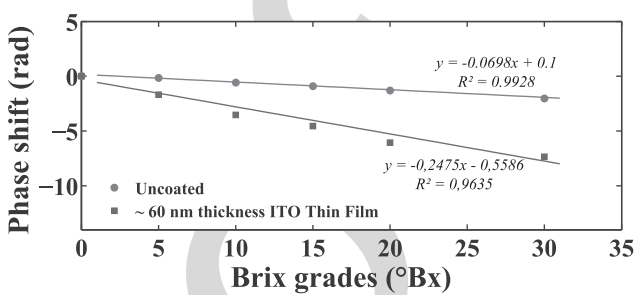

(b)

Fig. 16. Phase shift of a brix grades sensor: (a) Without ITO thin-film; (b) with $60 \mathrm{~nm}$ thickness ITO thin-film.

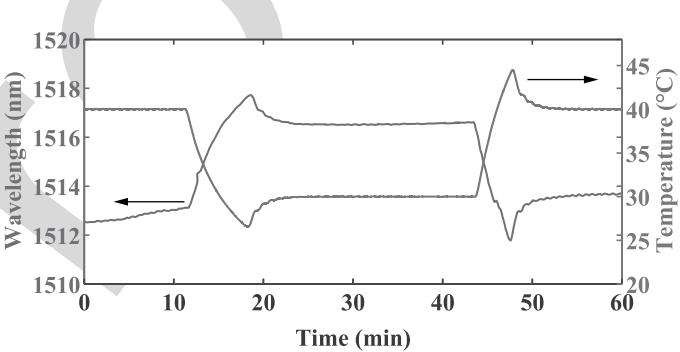

(a)

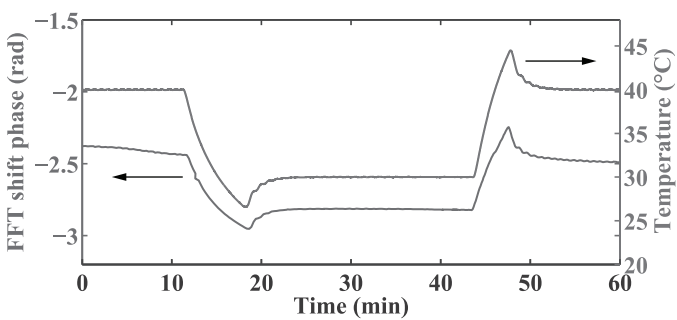

(b)

Fig. 17. (a) Wavelength shift and (b) phase shift temperature cross sensitivity.

\section{E. Temperature Cross Sensitivity}

Fig. 17(a) and (b) show the behavior of the wavelength shift 252 of an attenuation band and the phase shift with the temperature 253 setting as described in Section II-E. This probe was realized 254 with the $60 \mathrm{~nm}$ thin-film thickness sensor. The phase behavior 255 observed followed a trend imposed by the temperature's profile 256 generated as a consequence of the set points established. The 257 sensitivity was $0.3 \mathrm{~nm} /{ }^{\circ} \mathrm{C}$ and $0.034 \mathrm{rad} /{ }^{\circ} \mathrm{C}$.

A $0.14{ }^{\circ} \mathrm{Bx} /{ }^{\circ} \mathrm{C}$ of sensitivity was calculated for the ITO thinfilm coated sensors analyzed in the previous section.

\section{CONCLUSION}

This manuscript presented the optimization of SMS fiber 262 structures with a combined application of two techniques: etch- 263 ing and deposition of a thin-film. By an adequate design it 264 was possible to track both wavelength shifts of the optical spec- 265 trum and, by applying a simple FFT measurement technique, the 266 
phase shift of the fundamental frequency. The FFT measurement used in the analysis is an easy method that can be most applicable to networks that require narrow band, multiplexing capability and that have some problems related with high losses and noise.

The results also showed that the sensitivity obtained for this configuration of SMS was enhanced by reduction of the fiber diameter and by increasing the ITO film thickness. A good agreement was achieved between the experimental and the simulated approaches for this sensing device. A sensitivity of 1442 $\mathrm{nm} / \mathrm{RIU}$ was obtained by tracking the wavelength shift in a SMS with $25 \mu \mathrm{m}$ diameter and a $60 \mathrm{~nm}$ ITO thickness film, whereas for the same device, the FFT phase shift analysis showed a $0.24 \mathrm{rad} /$ RIU sensitivity.

These sensitivities, which are in the order of magnitude of other structures such as long period fiber gratings (LPFGs) (but with an inherently simpler manufacture process), place the developed sensing device as a good option for applications where high sensitivities and compact structures are required. As an example, a degrees Brix sensor has been presented, where the deposition of an ITO thin-film enhances the sensitivity of the device by a factor of 3 .

[1] C. R. Dennison, P. M. Wild, D. R. Wilson, and M. K. Gilbart, “An in-fiber Bragg grating sensor for contact force and stress measurements in articular joints," Meas. Sci. Technol., vol. 21, no. 11, 2010, Art. no. 115803.

[2] I. Del Villar, I. R. Matias, F. J. Arregui, and M. Achaerandio, "Nanodeposition of materials with complex refractive index in long-period fiber gratings," J. Lightw. Technol., vol. 23, no. 12, pp. 4192-4199, Dec. 2005.

[3] R. Kashyap, Fiber Bragg Gratings. New York, NY, USA: Academic, 1999.

[4] F. Chiavaioli et al., "Sol-gel-based titania-silica thin film overlay for long period fiber grating-based biosensors," Anal. Chem., vol. 87, no. 24, pp. 12024-12031, 2015.

[5] I. Del Villar, C. R. Zamarreño, M. Hernaez, F. J. Arregui, and I. R. Matias, "Lossy mode resonance generation with indium-tin-oxide-coated optical fibers for sensing applications," J. Lightw. Technol., vol. 28, no. 1, pp. 111-117, Jan. 2010.

[6] J. Homola, "Surface plasmon resonance sensors for detection of chemical and biological species," Chem. Rev., vol. 108, no. 2, pp. 462-493, 2008.

[7] Y. Cardona Maya, N. Gómez Cardona, and P. I. Torres Trujillo, "Low cost heat-and-pull rig for manufacturing adiabatic optical fiber tapers," Revista Facultad de Ingenieria Universidad de Antioquia, no. 70, pp. 167-172, 2014. [Online]. Available: http://aprendeenlinea.udea.edu.co/revistas/ index.php/ingenieria/article/viewFile/14820/16007

[8] T. Zhu, D. Wu, M. Liu, and D.-W. Duan, "In-line fiber optic interferometric sensors in single-mode fibers," Sensors, vol. 12, no. 8, pp. 10430-10449, 2012

[9] I. D. Villar, A. B. Socorro, J. M. Corres, F. J. Arregui, and I. R. Matias, "Refractometric sensors based on multimode interference in a thin-film coated single-mode-multimode-single-mode structure with reflection configuration," Appl. Opt., vol. 53, no. 18, pp. 3913-3919, 2014.

[10] Q. Wang and G. Farrell, "All-fiber multimode-interference-based refractometer sensor: Proposal and design," Opt. Lett., vol. 31, no. 3, pp. 317319, 2006.

[11] J. Lou, L. Tong, and Z. Ye, "Modeling of silica nanowires for optical sensing," Opt. Express, vol. 13, no. 6, pp. 2135-2140, 2005.

[12] A. W. Snyder and J. Love, Optical Waveguide Theory. Berlin, Germany: Springer, 2012.

[13] P. R. Cooper, "Refractive-index measurements of liquids used in conjunction with optical fibers," Appl. Opt., vol. 22, no. 19, pp. 3070-3072, 1983.

[14] L. Hoyt, "New table of the refractive index of pure glycerol at $20 \mathrm{C}$," Ind. Eng. Chem., vol. 26, no. 3, pp. 329-332, 1934.

[15] I. Malitson, "Interspecimen comparison of the refractive index of fused silica*," J. Opt. Soc. Amer, vol. 55, no. 10, pp. 1205-1209, 1965.

[16] D. Barrera et al., "Low-loss photonic crystal fiber interferometers for sensor networks," J. Lightw. Technol., vol. 28, no. 24, pp. 3542-3547, Dec. 2010.
[17] D. Leandro, M. Bravo, A. Ortigosa, and M. Lopez-Amo, "Real-time FFT analysis for interferometric sensors multiplexing," J. Lightw. Technol. vol. 33, no. 2, pp. 354-360, Jan. 2015.

[18] P. Wang, G. Brambilla, M. Ding, Y. Semenova, Q. Wu, and G. Farrell, "High-sensitivity, evanescent field refractometric sensor based on a tapered, multimode fiber interference," Opt. Lett., vol. 36, no. 12, pp. 2233 2235,2011

[19] I. Del Villar, J. L. Cruz, A. B. Socorro, J. M. Corres, and I. R. Matias, "Sensitivity optimization with cladding-etched long period fiber gratings at the dispersion turning point," Opt. Express, vol. 24, no. 16, pp. 1768017685, 2016.

[20] F. Chiavaioli et al., "Towards sensitive label-free immunosensing by means of turn-around point long period fiber gratings," Biosensors Bioelectron., vol. 60, pp. 305-310, 2014.

Yamile Cardona-Maya received the degree in engineering physics and the M.Sc. degree in physics from the Universidad Nacional de Colombia, Medellin, Colombia, in 2011 and 2014, respectively. She is currently working toward the Ph.D. degree in sciences-physics. In 2016, she was a visiting Ph.D. student at the Public University of Navarre, Pamplona, Spain. Her research is focused on optical fiber sensors for applications in life sciences and different industries branches.

Ignacio Del Villar received the M.S. degree in electrical and electronic engineering and the $\mathrm{Ph} . \mathrm{D}$. degree, specialty in optical fiber sensors, from the Public University of Navarra, Pamplona, Spain, in 2002 and 2006, respectively.

During 2004, he was a Visiting Scientist at the Institute d'Optique, Orsay, France, and in 2005, he was a Visiting Scientist in the Department of Applied Physics, University of Valencia, Burjassot, Spain. His research interests include optical fiber sensors and the analysis of waveguides and nanostructured materials, where he has coauthored more than 100 chapter books, journals, and conference papers. He is currently a reader at the Public University of Navarra since 2008, an Associate Editor of the Optics \& Laser Technology Journal since 2012, and an Associate Editor of the Journal of Sensors since 2014.

Abian B. Socorro received the M.Sc. degree in biomedical engineering and the Ph.D. degree in engineering both from the Public University of Navarre (PUNUPNA), Pamplona, Spain, in 2012 and 2015, respectively. Since 2010, he has been an Electrical and Electronic Engineer. In 2014, he was a visiting Ph.D. student in the Armani Research Laboratory, University of Southern California, Los Angeles, CA, USA. He is currently working as an Assistant Professor at the PUN-UPNA. His research interests include fiber-optic biosensors, fiber in the body, surface biofunctionalization, and other biomedical engineering applications.

Jesus M. Corres received the M.S. degree in electrical engineering and the Ph.D degree both from the Public University of Navarra (UPNA), Pamplona, Spain, in 1996 and 2003, respectively. He is currently working as an Associate Professor in the Department of Electrical and Electronic Engineering, UPNA. He is the author or coauthor of more than 100 publications. His main research interest include the development of fiber-optic sensors using nanostructured materials for biomedical, environmental, and safety applications. He is an Associate Editor of the IEEE SENSOR LETTERS and Hindawi Journal of Sensors.

Ignacio R. Matias received the M.S. degree in electrical and electronic engineering and the Ph.D. degree in optical fiber sensors from the Polytechnic University of Madrid, Madrid, Spain, in 1992 and 1996, respectively. In 1996, he became a Lecturer at the Public University of Navarra, Pamplona, Spain, where currently he is a permanent Professor. He has coauthored more than 300 chapter books, journals, and conference papers related to optical fiber sensors and passive optical devices and systems. He is a Senior and Topical Editor of the IEEE SENSORS JOURNAL.

Juan F. Botero-Cadavid was born in Colombia in 1979. He received the B.S and M.S. degrees in mechanical engineering and physics, from the Universidad Nacional de Colombia, Medellin, Colombia, in 2004 and 2007, respectively, and the Ph.D. degree in mechanical engineering from the University of Victoria, Victoria, BC, Canada, in 2014.

Since 2008, he has been an Assistant Professor in the School of Physics, Universidad Nacional de Colombia. His research interests include, but are not limited to, mechanical design, optical fiber sensors, digital holography, machining, and polymer electrolyte fuel cells. 